\title{
Bärentraube und Goldrutenkraut effektiv bei Blasenentzündung
}

_ Bereits im Mittelalter finden sich in englischen Heilpflanzenbüchern Hinweise auf die positive Wirkung der Echten Bärentraube (Arctostaphylos uva-ursi) bei Harnwegsinfektionen. Die Wirksubstanzen wurden im Laufe der Zeit genauer untersucht und in den 1950er-Jahren wurde Arbutin als zentraler Wirkstoff klassifiziert. 1955 kam das erste Fertigarzneimittel mit dem Extrakt der getrockneten Bärentraubenblätter (Uvae ursi folium) auf den Markt. Seit 1990 sind in Deutschland Cystinol akut ${ }^{\circledR}$ Dragées zur Behandlung von Blasenentzündungen erhältlich. Der standardisierte Bärentraubenblättertrockenextrakt hat gegenüber Tee den Vorteil, dass jedes Dragée die gleiche, hoch dosierte Wirkstoffmenge enthält. 1994 wurde die Wirksamkeit der Bärentraubenblätter bei entzündlichen Erkrankungen der ableitenden Harnwege durch die Monografie des damaligen Gesundheitsamtes offiziell bestätigt. Offizielle Anerkennungen durch die Monografien der WHO (2002) und ESCOP (2003) folgten. Bei unkomplizierten Harnwegsinfekten kann die Behandlung mit einem Bärentrauben-Präparat Antibiotika überflüssig machen.

Bei jeder vierten Frau, die einmal eine Blasenentzündung hatte, entwickelt sich daraus ein rezidivierender Harnwegsinfekt. In solchen Fällen kann der Trockenextrakt aus Echtem Goldrutenkraut (Cystinol long ${ }^{\circledR}$ Kapseln) hilfreich sein. Das Echte Goldrutenkraut wirkt durchspülend, entzündungshemmend (an der Blasenschleimhaut) und krampflösend. Dank dieses breiten Wirkspektrums können Präparate mit Echter Goldrute sowohl zur Vorbeugung von Blasenentzündungen als auch als Begleittherapie angewendet werden.

\section{Nach Informationen von}

Schaper \& Brümmer, Salzgitter-Ringelheim

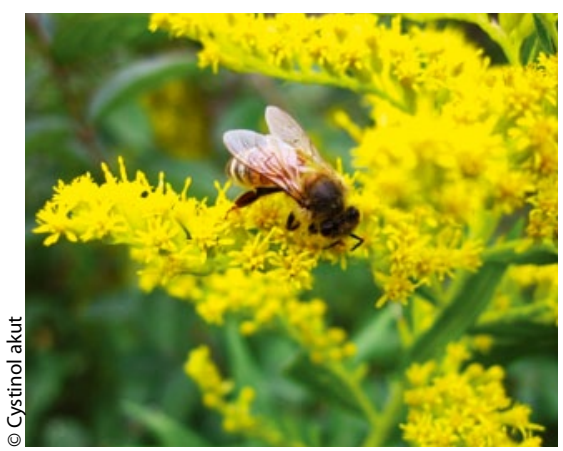

Goldrutenkraut kann bei rezidivierenden Harnwegsinfekten hilfreich sein.

\section{Selbstkatheterismus bei MS-Patienten für mehr Lebensqualität}

\begin{abstract}
— Neurogene Blasenentleerungsstörungen hindern Multiple-Sklerose-Patienten (MS) oft bereits im Frühstadium der Erkrankung an einem selbstbestimmten Alltag. Der intermittierende Selbstkatheterismus (ISK) ist in vielen Fällen die Anwendung der ersten Wahl. Voraussetzung: Patienten benötigen sensible Hinführung und Erklärung, aber auch regelmäßige Beratung.

„Die Erfahrung hat uns gezeigt, dass MS-Patienten großen Bedarf an Beratung hinsichtlich der alltagstauglichen Behandlung von neurogenen Blasenentleerungsstörungen haben", erklärt Thomas Haslinger, Geschäftsführer von PubliCare. „Diesem Bedarf werden wir explizit entsprechen." So wird beispielsweise für Erstgespräche die benötigte Zeit eingeplant, um MS-Patienten in aller Ruhe die
\end{abstract}

Selbstkatheterisierung zu erklären. Haslinger ergänzt:„Es ist ein intimes Thema, welches im häuslichen Umfeld perfekt aufgehoben ist, zumal unsere Patienten hier unter realen Bedingungen üben - nämlich auf der eigenen Toilette." Als herstellerunabhängiger Nachversorger kümmert sich PubliCare auch um die Neueinstellung.

Haslinger schätzt, dass derzeit noch etwa $30 \%$ der niedergelassenen Urologen ihren Patienten mit neurogener Blasenentleerungsstörung einen verbleibenden Katheter verordnen: „Wir wollen künftig noch mehr aufklären und für die alltagstaugliche Selbstkatheterisierung schulen und so für mehr Lebensqualität der MS-Patienten sorgen."

Nach Informationen von PubliCare, Köln

\section{Neues Antiinfektivum auf dem Markt}

Ab sofort steht neu das Präparat Fluomizin ${ }^{\circledR} 10 \mathrm{mg}$ Vaginaltabletten zur lokalen Behandlung der bakteriellen Vaginose zur Verfügung. Jede Vaginaltablette enthält $10 \mathrm{mg}$ Dequaliniumchlorid. Fluomizin ${ }^{\oplus}$ von der Firma Pierre Fabre Pharma ist in der therapeutischen Packungsgröße mit sechs Vaginaltabletten erhältlich.

Das Antiinfektivum Fluomizin ${ }^{\circledR}$ ist ein lokales Arzneimittel gegen bakterielle Vaginose, das auch in allen Phasen der Schwangerschaft verwendet werden kann.

In einer Vergleichsstudie gegen den sogenannten. "Goldstandard“, das Antibiotikum Clindamycin, konnte nachgewiesen werden, dass Fluomizin ${ }^{\circledR}$ dieselbe klinische Wirksamkeit besitzt. Im Follow-up nach zwei bis sechs Wochen zeigte sich sogar eine signifikant bessere Heilungsrate.

Nach Informationen von

Pierre Fabre, Freiburg

Mito-medac ${ }^{\circledast}$ im AOK-Rabattvertrag

Am 1. Juni 2011 ist der AOKRabattvertrag für Mitomycin gestartet, in dem medac bundesweit exklusiver Partner der AOK ist. Mit diesem jüngsten Rabattvertrag der medac profitieren inzwischen rund 30 Millionen Versicherte von einer preisgünstigen Versorgung mit mito-medac ${ }^{\circledast}$.

Das Präparat ist zugelassen für die intravesikale Anwendung zur Rezidivprophylaxe bei oberflächlichem Harnblasenkarzinom nach transurethraler Resektion.

Eine Übersicht über alle bestehenden Rabattverträge zu mito-medac ${ }^{\circledR}$ können Ärzte anfordern unter der Telefonnummer (0 41 03) 8006 - 321.

Nach Informationen von

Medac, Wedel 